\title{
Towards Inhibition of Vif-APOBEC3G Interaction: Which Protein to Target?
}

\author{
Iris Cadima-Couto and Joao Goncalves \\ URIA-Centro Patogénese Molecular and Instituto de Medicina Molecular, Faculdade de Farmácia da Universidade Lisboa, \\ Avenue Das Forcas Armadas, 1649-019 Lisboa, Portugal \\ Correspondence should be addressed to Joao Goncalves, joao.goncalves@ff.ul.pt
}

Received 2 May 2010; Revised 31 July 2010; Accepted 14 August 2010

Academic Editor: Michael Bukrinsky

Copyright ( 92010 I. Cadima-Couto and J. Goncalves. This is an open access article distributed under the Creative Commons Attribution License, which permits unrestricted use, distribution, and reproduction in any medium, provided the original work is properly cited.

\begin{abstract}
APOBEC proteins appeared in the cellular battle against HIV-1 as part of intrinsic cellular immunity. The antiretroviral activity of some of these proteins is overtaken by the action of HIV-1 Viral Infectivity Factor (Vif) protein. Since the discovery of APOBEC3G $(\mathrm{A} 3 \mathrm{G})$ as an antiviral factor, many advances have been made to understand its mechanism of action in the cell and how Vif acts in order to counteract its activity. The mainstream concept is that Vif overcomes the innate antiviral activity of A3G by direct protein binding and promoting its degradation via the cellular ubiquitin/proteasomal pathway. Vif may also inhibit A3G through mechanisms independent of proteasomal degradation. Binding of Vif to A3G is essential for its degradation since disruption of this interaction is predicted to stimulate intracellular antiviral immunity. In this paper we will discuss the different binding partners between both proteins as one of the major challenges for the development of new antiviral drugs.
\end{abstract}

\section{Introduction}

Vif is a $23-\mathrm{kDa}$ cytoplasmic protein that is expressed from a partially spliced mRNA in Rev-dependent manner during the late phase of HIV-1 replication. The human immunodeficiency virus type 1 (HIV-1) Vif protein is essential for virus replication in primary lymphoid and myeloid cells, but is dispensable for efficient replication in several transformed T-cell lines as well as in nonlymphoid cell lines such as HeLa and 293T [1-3]. Cells that are unable to support the replication of Vif-defective HIV-1 (HIV-1 $\triangle$ Vif) have been termed "nonpermissive," while cells that can sustain HIV$1 \triangle$ Vif replication are termed "permissive."

These findings were made over 15 years ago but the molecular mechanisms underlying this cell-specific difference were maintained a mystery until 1998. The observation that heterokaryons formed by fusion of nonpermissive and permissive cells exhibit the nonpermissive phenotype [4, 5] led to the hypothesis that nonpermissive cells express an inhibitor of HIV-1 replication that is blocked by the viral Vif protein. Later, in 2002, Sheehy et al. reported the identification of the apolipoprotein B mRNA-editing enzyme-catalytic polypeptide-like 3G (APOBEC3G) as the HIV-1 replication inhibitor [6].

A3G is located in the cytoplasm of the cell and has been shown to be an exclusive DNA mutator [7]. A3G exists either as enzymatically active low-molecular-mass (LMM) forms consistent with enzyme monomers or dimers, or as an enzymatically inactive high-molecular-mass (HMM) ribonucleoprotein complex larger than $2 \mathrm{MDa}$ [8]. LMM $\mathrm{A} 3 \mathrm{G}$ is found in resting CD4+ T cells of peripheral blood and macrophages where it may act as a powerful antiviral restriction factor for HIV-1 [8]. Conversely, resting CD4+ T cells in lymphoid tissues are permissive to HIV-1 infection as $\mathrm{A} 3 \mathrm{G}$ is expressed predominantly in HMM complexes due to the lymphoid microenvironment [9]. It was reported that in lymphoid tissues, cytokines such as IL-2 and IL-5 are responsible for the stimulation of HMM complexes, which in turn may confer the permissive phenotype for HIV-1 infection [9]. Nonetheless, only one research group have provided data to support a role for APOBEC3G in restriction of HIV-1 in quiescent CD4+ T cells. Therefore, to date the primary mechanism of HIV-1 restriction in quiescent CD4+ $\mathrm{T}$ cells remains to be elucidated. 


\section{The APOBEC3 Family}

A3G belongs to a family of polynucleotide cytidine deaminases (CDAs), whose members include seven family members, named APOBEC3A to $\mathrm{H}(\mathrm{A} 3 \mathrm{~A}-\mathrm{H})$. All of these genes are clustered on chromosome $22[10,11]$. During mammalian evolution APOBEC3 (A3) family members have evolved from a single gene in mice, located on chromosome 15 , to eight genes $(\mathrm{A} 3 \mathrm{~A}-\mathrm{H})$ in primates $[10,11]$. Interestingly, expansion of the A3 gene cluster contrasts with the decline in retrotransposition activity in primates [10-12]. This observation raises the possibility that APOBEC 3 genes may have evolved to prevent genomic instability caused by endogenous retroelements [13].

All members of the $\mathrm{A} 3$ family contain one (A3A, A3C, $\mathrm{A} 3 \mathrm{H})$ or two (A3B, A3DE, A3F, A3G) copies of a conserved zinc-binding motif, His/Cys-X-Glu-X $23-28$-Pro-Cys- $\mathrm{X}_{2-4^{-}}$ Cys, that catalyzes the hydrolytic deamination at the $\mathrm{C} 4$ position of $2^{\prime}$-deoxycytidine, resulting in a $2^{\prime}$-deoxyuridine $[6,10,11,14-16]$. These proteins also contain a key glutamate required for proton shuttling during catalysis and two key aromatic residues involved in RNA substrate binding [16-22].

Proteins of the $\mathrm{A} 3$ family protect cells against the invasion of a variety of viruses $[6,23-25]$. Of these, $A 3 G$ and A3F are the most extensively studied due to their strong activity against HIV-1 [26]. Like A3G and A3F, A3B and A3DE contain two CDAs and have been shown to display moderate antiviral activity [23, 27-29]. Despite having only one CDA domain $\mathrm{A} 3 \mathrm{~A}, \mathrm{~A} 3 \mathrm{C}$, and $\mathrm{A} 3 \mathrm{H}$ also impair HIV-1 infection [30-33].

The interplay between HIV-1 proteins and host restriction factors, such as $\mathrm{A} 3 \mathrm{G}$ and $\mathrm{A} 3 \mathrm{~F}$ are potential targets for the development of new antiviral drugs. Inhibition of this interplay could allow the host innate defences to control viral replication. The interaction between Vif and A3G proteins has been extensively studied in the last decade and several regions and amino acid residues have been described as involved in the interaction between these two proteins.

In this paper we will briefly describe the current knowledge on the different binding strategies between Vif and $\mathrm{A} 3 \mathrm{G}$, and discuss these mechanisms with the purpose of developing new antiviral drugs.

\section{APOBEC3 Restriction of HIV-1 Infection}

Expression of A3 proteins in HIV-1 infected cells can lead to their encapsidation into progeny virions through recruitment to viral or transposon capsid structures, probably involving Gag proteins and viral RNA [29, 34-37]. Deaminases will be delivered to the target cell where they will deaminate cytidines to uridines during the synthesis of the minus-strand viral cDNA [38, 39]. Consequently, during the synthesis of the plus-strand DNA, adenosines are incorporated instead of the original guanines resulting in G-to-A substitution. This process of deamination that will result in the loss of genetic integrity and protein function is commonly referred as hypermutation [40-42]. However, there is increasing evidence that $\mathrm{A} 3 \mathrm{G}$ is able to restrict HIV-1 infection in the absence of deaminase activity $[8,43-45]$.

A study carried by Newman et al. showed that certain amino acid substitutions in the C-terminal cytidine deaminase "core" domain of A3G (APOBEC3G has two such domains) originated mutant proteins that were unable to mutate DNA, yet maintained the antiviral activity [44]. However, cytidine deaminase (CDA) independent effects cannot be observed when the mutant proteins are expressed at physiological levels [46], making these nonenzymatic A3G effects somewhat controversial.

On the other hand, when unstimulated $\mathrm{CD} 4+\mathrm{T}$ cells (where $A 3 G$ is expressed as an active LMM form) were treated with A3G-specific small interfering RNAs (siRNA), the early replication block encountered by HIV-1 was greatly relieved [6]. When HIV-1 reverse transcripts in resting primary CD4 T cells were examined for evidence of A3Ginduced $\mathrm{dG} \rightarrow \mathrm{dA}$ hypermutations, only $8 \%$ of the transcripts contained such mutations suggesting an antiretroviral activity independent of deoxycytidine deaminase activity [8].

Additional studies indicated that initiation of HIV reverse transcription and/or processivity of reverse transcriptase (RT) could be inhibited by A3G [47-52]. Sequence analyses of 2-LTR circle junctions from unintegrated DNA synthesized in the presence of $\mathrm{A} 3 \mathrm{G}$ showed that the U5 end DNA occasionally had additional six RNA bases derived from the 3'-terminus of tRNALys3 [53]. These results suggest that A3G causes a defect during tRNA removal that limits plusstrand transfer and consequently affect viral DNA ends that will not be able to efficiently integrate into the host cell genomic DNA [53]. The process of successive inhibition of reverse transcription mechanisms has a cumulative effect and could explain end result of reducing viral integration. However, the decrease in plus-strand DNA transfer may not explain all the effects on viral cDNA synthesis by A3G [54]. Indeed, $\mathrm{A} 3 \mathrm{G}$ could be coimmunoprecipitated with $\mathrm{NC}$ and integrase (IN) in HIV-1 Vif-positive viruses [55]. In addition, A3F co-immunoprecipitation with virion-associated integrase (IN) was also observed [55]. Nevertheless, GSTpull down assays do not show binding between $\mathrm{A} 3 \mathrm{G}$ and RT, suggesting that interactions between $\mathrm{A} 3 \mathrm{G}$ and viral proteins may may inhibit the process of reverse transcription [49].

\section{APOBEC3 Restriction by Vif}

To overcome the antiviral effect of APOBEC3 proteins, in particular A3G and A3F, HIV-1 encode the Vif protein. The mode of action by which Vif counteracts A3G and A3F-mediated antiviral activity has been extensively studied. Vif neutralizes the antiviral activity of A3G and A3F by forming a RING-finger E3 ubiquitin complex with Elongin $\mathrm{B}$ (EloB) and C (EloC), Cullin 5 (Cul5) and Ring-box protein 2 (Rbx2) (Figure 1(a)). By bringing A3G into contact with the RING-finger E3 ubiquitin complex, Vif promotes A3G polyubiquitination and its degradation in the $26 \mathrm{~S}$ proteasome [56-63]. A more a recent report suggested that A3G needs Vif polyubiquitination to be degraded rather than its own polyubiquitination, [64], but this is still a matter of debate. Moreover, Vif has also been reported to directly block 


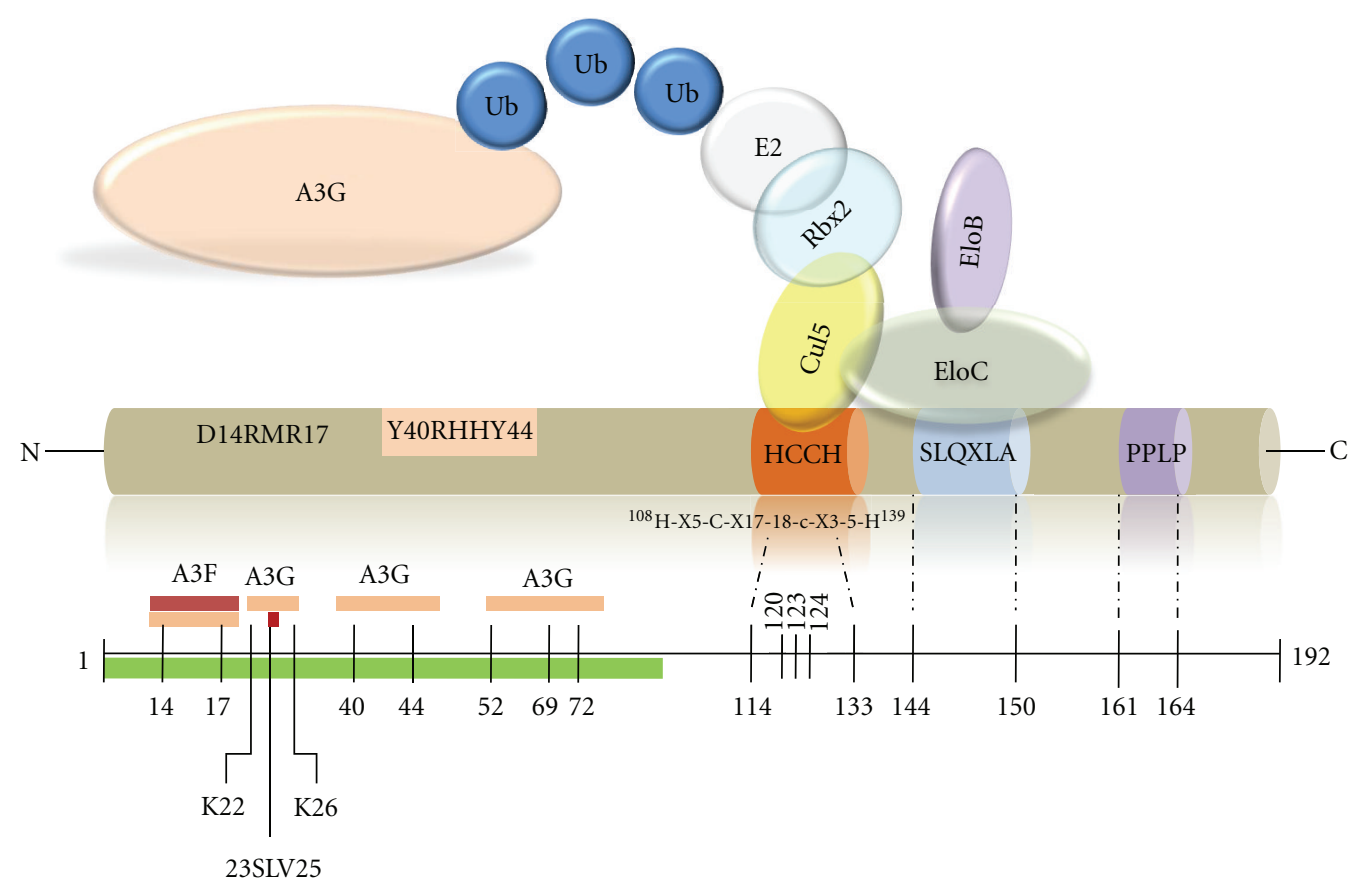

(a) HIV-1 Vif
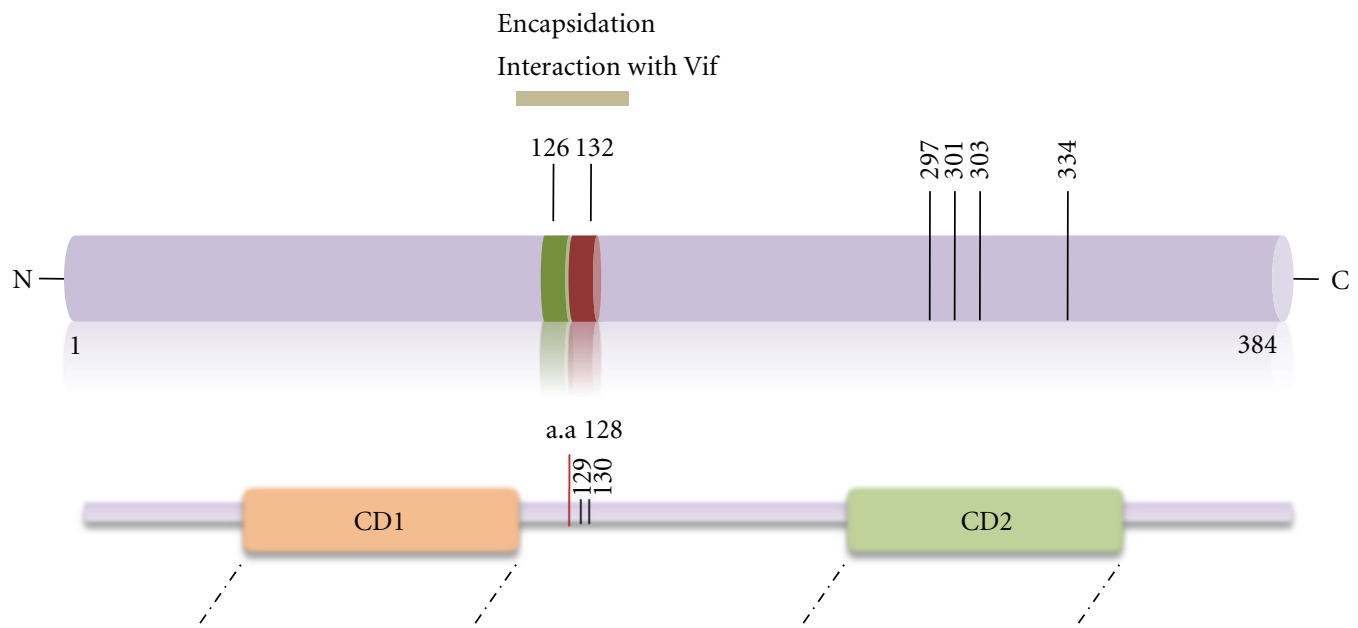

His/Cys-X-Glu- $\mathrm{X}_{23-28}$-Pro-Cys- $\mathrm{X}_{2-4}$-Cys

His/Cys-X-Glu- $\mathrm{X}_{23-28}$-Pro-Cys- $\mathrm{X}_{2-4}$-Cys

- Virion incorporation (a.a 105 - 156)

- RNA binding

- Deaminase activity

- Multimerization

- Substrate specificity

(b) Human APOBEC3G

FIGURE 1: Schematic representation of Vif and A3G domains involved in the interaction of both proteins. (a) Vif binds A3G through specific residues located in the N-terminal region. Amino acids in Vif that are involved in the interaction with A3G are shown in pink. C-terminal Vif domains involved in targeting A3G for proteasomal degradation are shown in orange (zinc binding HCCH domain), and light blue (SLQXLA). The multimerization domain is purple. (b) The catalytic domains (CD1 and CD2) and Vif-binding regions of A3G protein are represented. Amino acids 126-132 are involved in A3G encapsidation and interaction with Vif and are represented in green and red. 
A3G encapsidation $[57,65,66]$, reduce A3G translation $[60$, 65], and directly inhibit the catalytic activity of A3G [45]. It is still unknown whether all these mechanisms must operate in concert to inhibit A3G action. However, independently on the mode of action, the ultimate goal of Vif is to prevent A3G encapsidation into budding HIV-1 virions.

\section{The Vif-A3G Interaction}

Recent advances on the biological role of HIV-1 Vif and A3 proteins, together with progress in deciphering how Vif counteracts $\mathrm{A} 3 \mathrm{G}$ and $\mathrm{A} 3 \mathrm{~F}$ opened new opportunities to develop anti-HIV drugs. However, understanding the mode of action of Vif and A3G alone can provide a number of attractive targets for drug development since A3G displays the most potent activity against HIV-1.

Disruption of Vif-A3G interaction is predicted to rescue $A 3 G$ expression and virion packaging, consequently stimulating intracellular antiviral activity. Similarly, pharmacologic studies to suppress A3G proteasome-mediated degradation have been shown to enhance A3G half life and consequently inhibit HIV-1 infection [59]. In order to facilitate the rational design of inhibitors for Vif-A3G interaction, experimental assays have been devised to define features of Vif that are involved in the interaction with A3G, and vice versa.

The N-terminal region of HIV-1 Vif is important for binding and neutralization of $\mathrm{A} 3 \mathrm{G}$ and $\mathrm{A} 3 \mathrm{~F}$ and also contributes to species-specific recognition $[58,67-70]$. In the C-terminal region of Vif, the highly conserved cysteine residues at positions 114 and 133 and the S $^{144}$ LQXLA $^{149}$ motif (Figure 1(a)) are required for Vif function and HIV-1 replication [71, 72]. Vif associates with the Cul5-EloB-EloC complex by directly binding to EloC via a BC box motif at positions 144 to 150 and to Cul5 via hydrophobic residues at positions 120,123, and 124 within a zinc-binding region formed by the highly conserved HCCH motif (Figure 1(a)) $[73,74]$. The SLQXLA motif is essential for targeting A3G for proteasomal degradation. Substitution of the SLQ portion of the SLQXLA motif has been reported to be sufficient to prevent A3G degradation [57, 59, 75]. The zinc bindingmotif $\mathrm{HCCH}$ is also involved in $\mathrm{A} 3 \mathrm{G}$ degradation and necessary for the specificity of Vif-Cul5 interaction [76, 77].

Several groups have shown that Vif-induced degradation of $\mathrm{A} 3 \mathrm{G}$ requires the physical interaction of the two proteins and that a single amino acid change in $\mathrm{A} 3 \mathrm{G}$ at residue 128 was sufficient to abolish this interaction [78-80]. This assumption led to the conclusion that the Vif-A3G interaction is species-specific and is determined by aspartic acid at position 128 in $\mathrm{A} 3 \mathrm{G}$ and lysine in African Green Monkey (AGM) [78-81]. Substitution of human A3G D128 by $\mathrm{K} 128$, found in AGM A3G, results in a mutant (D128KA3G) protein that is resistant to the effect of Vif. This data can be explained either because the mutant protein is no longer able to interact with Vif or due to inhibition of subsequent downstream steps [68, 78, 79, 81].

Experiments using alanine-scanning and multiple synonym substitutions on $\mathrm{A} 3 \mathrm{G}$ residues confirmed the central role played by the aspartic acid at position 128 and showed the crucial role of proline-129 and aspartic acid-130, as important contributory residues (Figure 1(b)) [82].

Specifically, resistance to Vif induced degradation was conferred by mutating the aspartic acid residue at position 128 or 130 to the positively charged residue lysine, indicating that the interaction between Vif and A3G is largely determined by electrostatic interactions involving these residues [82]. However, residue 128 has been shown to be more sensitive to amino acid alterations than 130, suggesting that amino acid D128 may play a more prominent role in A3G interaction with Vif [82].

Substitution of proline in residue 129 of A3G to alanine or glycine displayed a strong Vif-resistant phenotype indicating that a specific structural interaction is also required for an efficient inhibition of A3G by Vif [82]. In addition to electrostatic determinants, one study reported that $\mathrm{A} 3 \mathrm{G}$ residues 54-124 were sufficient to coimmunoprecipitate Vif, suggesting the role of additional interacting domains between Vif and A3G [56]. Another study reported that amino acids 105-156 of A3G were sufficient for its interaction with Vif, and amino acids 157-245 were required for its degradation [83]. Recently, analysis of A3G chimeras identified amino acids $126-132$ as critical determinants involved in Vif interaction (Figure 1(b)) [84]. Finally, by using model-guided mutagenesis, four Lys residues in the CDA of A3G (Lys-297, 301, 303, and 334) were recently identified as required for Vif-mediated polyubiquitination and degradation (Figure 1(b)) [85].

Asparagine at position 128 of $\mathrm{A} 3 \mathrm{G}$ was shown to interact with amino acids 15 or 17 of Vif, and mutations in the $\mathrm{D}^{14} \mathrm{RMR}^{17}$ conserved region of Vif can also alter its speciesspecific effect [68]. Alteration of DRMR region to SERQ or SEMQ, which are present in SIV $_{\mathrm{AGM}}$ Vif, promotes the interaction of AGM A3G, rhesus (Rh) A3G, and D128KA3G with HIV-1 Vif [68]. The loss of species restriction is probably caused by an overall increase in the negative charge of amino acids in the 14-17 region of HIV-1 Vif, which promotes effective interaction with the positive charge of lysine present at residue 128 in AGM A3G and Rh A3G. In addition, the DRMR region was also shown to be critical for the binding strength between A3G and Vif [68] although, additional interaction motifs were required for stabilization of this interaction (Figure 1(a)) [86].

By performing an extensive mutational analysis of Vif, Russell and Pathak identified the new motif $\mathrm{Y}^{40} \mathrm{RHHY}^{44}$, that was shown to be involved in binding to A3G (amino acids 126-132) (Figure 1(a)) [86]. Vif $\mathrm{Y}^{40} \mathrm{RHHY}^{44}$ motif was considered a critical domain for binding to A3G while the $\mathrm{D}^{14} \mathrm{RMR}^{17}$ domain could be involved in a secondary step involving A3G degradation [86].

Nonetheless, other amino acids in Vif may also contribute to A3G binding. Mehle et al. demonstrated that amino acids 40 to 71 in the $\mathrm{N}$-terminus of Vif contain a nonlinear binding site for A3G and that His 42/43 are important for Vif-A3G binding and Vif-mediated degradation of $\mathrm{A} 3 \mathrm{G}$ in vivo [87]. Another region of Vif comprising amino acids 52 to 72 was identified as responsible for Vif-mediated degradation and virion exclusion of A3G (Figure 1(a)) [88]. 
Recently, a highly conserved ${ }^{69} \mathrm{YXXL}^{72}$ motif in Vif was shown to mediate the binding to human $\mathrm{A} 3 \mathrm{G}$ and its subsequent degradation (Figure 1(a)) [88-90]. Pery et al. showed that this motif was critical for in vitro direct binding of recombinant GST-Vif (1-94 a.a) to A3G and by FRET assay [89]. Additionally, Vif residues 22-26 and Y30 were also involved in the interaction with APOBEC3 proteins [91, 92]. In particular, Vif K22 and K26 were shown to be important for degradation of A3G. Additionally, residue Y30 was involved in the interaction with both A3G and $\mathrm{A} 3 \mathrm{~F}$ raising the hypothesis that alteration of Tyr in position 30 may affect the conformational stability of Vif. Although alanine-scanning of ${ }^{23} \mathrm{SLV}^{25}$ region did not reduce the ability of Vif to bind A3G and/or A3F, the antiviral effect was abolished [91, 92], reinforcing the idea that Vif binding to A3G does not necessarily lead to its degradation.

Vif-mediated degradation of $\mathrm{A} 3 \mathrm{G}$ is regulated by phosphorylation of Vif and A3G at Ser144 and Thr32, respectively $[61,93]$. It was recently documented that phosphorylation of A3G by protein kinase A (PKA) reduces its binding to Vif affecting subsequent ubiquitination and degradation [93]. This finding indicates that phosphorylation events may also play an important role in the interaction between Vif and APOBEC3 proteins.

The central hydrophilic domain, $\mathrm{E}^{88} \mathrm{WRKKR}^{93}$, and the proline-rich $\mathrm{P}^{161} \mathrm{PLP}^{164}$ domain (Figure $1(\mathrm{a})$ ) of Vif have been implicated in enhancing its steady-state level and in binding to tyrosine kinases, respectively [94, 95]. It is conceivable that the $\mathrm{E}^{88} \mathrm{WRKKR}^{93}$ motif is involved in maintaining sufficient intracellular levels of Vif necessary for A3G inhibition. Mutations in the PPLP motif of Vif were shown to reduce the infectivity of virions produced in $\mathrm{T}$ cells and inhibit Vif-Vif interaction in vitro [96]. It has been suggested that multimerization of Vif may be necessary for $A 3 G$ binding and PPLP region may be essential for this behaviour [97]. In addition, this region has also been identified as part of a novel "SOCS-box" motif implicated in binding to EloC $[61,62]$ and involved in the interaction of Vif with the cellular Hck tyrosine kinase [98, 99]. Recently, Donahue et al. demonstrated that mutations in PPLP motif impaired the ability of Vif binding to A3G, but did not affect EloC and Cullin5 binding [100].

Other additional studies have identified regions in Vif protein that are responsible for $\mathrm{A} 3 \mathrm{G}$ and $\mathrm{A} 3 \mathrm{~F}$ inhibition but are not located in the SLQXLA and HCCH motifs. As an example, Simon et al. reported that any single amino acid substitutions in Vif sequences isolated from HIV-1 infected patients were sufficient to prevent A3G neutralization [67].Moreover, a subset of mutants has been reported to be functional against $\mathrm{A} 3 \mathrm{G}$ but not $\mathrm{A} 3 \mathrm{~F}$ and vice versa [86].

\section{Conclusion Remarks}

To this date, the most successful HIV-1 antiviral drugs in the market are those that target the HIV enzymes reverse transcriptase (RT) and protease (PR). Nevertheless, other strategies have proven to be highly effective such as integrase inhibitors [101-103], and entry inhibitors like T20 and Maraviroc [104].

As reviewed above, recent advances in the study of the biological and biochemical role of Vif and A3G, together with progress in deciphering how Vif counteracts $A 3 G$, opened new opportunities to develop novel anti-HIV drugs.

Blocking the binding of Vif to A3G in vivo is certainly one of the most obvious therapeutic strategies. Several authors reported that Vif may function at multiple levels to prevent incorporation of $\mathrm{A} 3 \mathrm{G}$ into viral particles $[45,60$, $61,105]$. Therefore, preventing the binding of Vif to A3G may have two outcomes: (1) inhibition of $\mathrm{A} 3 \mathrm{G}$ proteasomal degradation and, (2) increasing in the amount of A3G at viral assembly locations, resulting in a higher level of A3G incorporation into virions. However, care must be taken when attempting to increase A3G intracellular levels as A3G mRNA is highly expressed in some tumour cells [106], and this may potentially induce tumour formation.

The amount of intracellular Vif can be reduced by degradation in the proteasome due to direct interaction with the SCF complex. Consequently, disruption of Vif-A3G interaction could prevent the proteolytic degradation of A3G and consequently increase the intracellular levels of Vif by impeding its destruction. Whether increasing amounts of Vif may rescue viral replication by other mechanisms besides A3G degradation is a matter of debate [45].

A detailed knowledge of the protein domains involved in this interaction is extremely important for the rational design of new antiviral drugs. To this date, several regions in Vif and A3G proteins have been mapped, and the effect on their interaction has been studied. Nevertheless, the threedimensional molecular structure of both proteins was not yet determined, and only their structure homology modelling was reported $[82,83]$.

As described above, the most important domains of A3G responsible for Vif interaction involve the asparagine at position 128 and its surrounding residues. The charge of D128 amino acid in A3G markedly influences the interaction with Vif indicating that the protein binding is dependent on electrostatic forces [68]. Thus, chemical compounds targeting this region could be effective in preventing VifA3G interaction. Importantly, molecules targeting amino acid 128 are not predicted to interfere with A3G enzymatic activity which is conferred by other domains of the protein $[107,108]$.

In addition to this region, other motifs in $A 3 G$ have been validated as potential targets for antiviral drugs. As previously discussed, other domains of A3G are involved in the mechanism of Vif binding. This conclusion probably reflects a broad structural interface that stabilizes Vif-A3G interaction. However, it is not known yet if these regions converge towards a unique functional structure since we lack a high-resolution structure of A3G. Therefore, the development of new drugs targeting D128 residue should take into account the involvement of multiple adjacent regions.

The Vif protein is also a potential target for HIV-1 drug therapy. In contrast to A3G, Vif has a scattered localization of motifs capable to mediate its interaction with APOBEC3 
proteins. Thus, it is conceivable that the mechanism by which Vif recognizes A3G may involve multiple and conserved functional structures in the viral protein. Whether these structures interact alone or in synergy towards A3G binding is not yet known.

The N-terminal region of Vif has been implicated in binding to $\mathrm{A} 3 \mathrm{G}$, whereas the protein motifs that mediate interaction with Cul5-E3 ligase complex and promote A3G degradation are concentrated in the C-terminal region of Vif $[58,67-70]$. Specifically, in the context of the ${ }^{144}$ SLQXLA $^{149}$ motif, lysine at position 145 is crucial for binding to EloC and to initiate the process of A3G-targeted degradation. Other regions, like the zinc-binding $\mathrm{HCCH}$ motif, and the Vif multimerization domain $\left({ }^{161} \mathrm{PPLP}^{164}\right)$ are also present in the C-terminus of Vif and may constitute alternative inhibition motifs. It has been shown that mutations in the ${ }^{161}$ PPLP 164 motif, reduced Vif binding and degradation of A3G without affecting the interaction of Vif with Elongin $C$ and Cullin5 [100]. Interestingly, when an anti-viral peptide that mimics the Vif PPLP dimerization domain was used, the amount of A3G incorporation into wild-type HIV-1 particles increased [109]. Thus, small molecules that target this motif could be developed as antiviral drugs to block the Vif-mediated inhibition of A3G and A3F activity.

Although the three-dimensional structure of HIV-1 Vif was not yet determined, a close perspective has been developed by comparative modelling [109]. Using molecular dynamics simulation, it was shown that mutations of critical residues led to the disruption of Vif and EloB-EloC interaction, consistent with experimental observations. These novel homology models of Vif can therefore provide structural information for investigating critical domains for protein neutralization.

While a complete and accurate structure is not available, antiviral drugs that could inhibit Vif and enhance A3G/A3F activity are emerging as attractive candidates [110-113]. For example, a small Vif antagonist that increases the intracellular level of A3G and its incorporation into virions in a Vif-dependent manner has been identified [114]. This compound was shown to enhance the degradation of Vif in an A3G-dependent manner without being a general inhibitor of the proteasome-mediated protein degradation [114].

Nonetheless, developing specific and effective small chemical inhibitors to directly inhibit Vif-A3G interaction faces many challenges due to the multiple binding regions involved. In addition, in vitro binding assays and cell-based assays that have been used to decipher the dynamic principles behind protein functional association make it sometimes difficult to assess the in vivo significance of the results. In particular, co-immunoprecipitation assays that have been commonly used to study specific domains involved in VifA3G interaction are questioned. One study has shown that Vif was able to inhibit virion encapsidation and the antiviral activity of an A3G degradation resistant mutant (C97A) [115], suggesting a direct inhibition of A3G by Vif. However, the authors could not rule out the possibility that VifA3G complexes could have been formed after cell lysis during co-immunoprecipitation assays. Finally, no one so far has been able to demonstrate a direct Vif-A3G interaction with purified components at physiological concentrations. Therefore, we cannot rule out the need for post-translational modifications or interactions during synthesis, or the need for additional components in the interaction complex.

It is plausible that the binding features of Vif-EloC interaction present mechanistic specificities that would be optimal for the rational development of a Vif antagonist. Moreover, the specific and stable interaction of ${ }^{144}$ SLQXLA ${ }^{149}$ domain of Vif with EloC will probably result in a better approach for drug screening.

However, attention must be taken when designing new antiviral drugs since incomplete Vif inhibition could result in increased $\mathrm{A} 3 \mathrm{G}$ levels in the cell enough to exert an intermediate level of mutational pressure on the HIV-1 genome resulting in a "sub-error catastrophe." This could accelerate viral evolution instead of inducing a population collapse resulting in anti-retroviral resistance [116] and immune escape. Despite some controversy remaining on that subject, a therapeutic strategy that could amplify this nonmutagenic phenotype without enhancing cytidine deamination may be an alternative to suppress viral replication. In addition, A3G and $\mathrm{A} 3 \mathrm{~F}$ may also function through other mechanisms that do not necessarily require deamination $[8,117,118]$.

In conclusion, antiviral drugs that could inhibit Vif and enhance $\mathrm{A} 3 \mathrm{G} / \mathrm{A} 3 \mathrm{~F}$ activity are emerging as attractive candidates [110-113]. Nevertheless, the potential outcome of a Vif-based intervention must be examined rigorously both in vitro and in vivo prior to clinical deployment.

\section{References}

[1] D. H. Gabuzda, K. Lawrence, E. Langhoff et al., "Role of vif in replication of human immunodeficiency virus type 1 in CD4+ T lymphocytes," Journal of Virology, vol. 66, no. 11, pp. 6489-6495, 1992.

[2] H. Sakai, R. Shibata, J.-I. Sakuragi, S. Sakuragi, M. Kawamura, and A. Adachi, "Cell-dependent requirement of human immunodeficiency virus type 1 vif protein for maturation of virus particles," Journal of Virology, vol. 67, no. 3, pp. 1663-1666, 1993.

[3] U. von Schwedler, J. Song, C. Aiken, and D. Trono, "vif is crucial for human immunodeficiency virus type 1 proviral DNA synthesis in infected cells," Journal of Virology, vol. 67, no. 8, pp. 4945-4955, 1993.

[4] N. Madani and D. Kabat, "An endogenous inhibitor of human immunodeficiency virus in human lymphocytes is overcome by the viral Vif protein," Journal of Virology, vol. 72, no. 12, pp. 10251-10255, 1998.

[5] J. H. M. Simon, N. C. Gaddis, R. A. M. Fouchier, and M. H. Malim, "Evidence for a newly discovered cellular anti-HIV-1 phenotype," Nature Medicine, vol. 4, no. 12, pp. 1397-1400, 1998.

[6] A. M. Sheehy, N. C. Gaddis, J. D. Choi, and M. H. Malim, "Isolation of a human gene that inhibits HIV-1 infection and is suppressed by the viral Vif protein," Nature, vol. 418, no. 6898, pp. 646-650, 2002.

[7] R. Suspène, P. Sommer, M. Henry et al., "APOBEC3G is a single-stranded DNA cytidine deaminase and functions independently of HIV reverse transcriptase," Nucleic Acids Research, vol. 32, no. 8, pp. 2421-2429, 2004. 
[8] Y.-L. Chiu, V. B. Soros, J. F. Kreisberg, K. Stopak, W. Yonemoto, and W. C. Greene, "Cellular APOBEC3G restricts HIV-1 infection in resting CD4+ T cells," Nature, vol. 435, no. 7038, pp. 108-114, 2005.

[9] J. F. Kreisberg, W. Yonemoto, and W. C. Greene, "Endogenous factors enhance HIV infection of tissue naive CD4 $\mathrm{T}$ cells by stimulating high molecular mass APOBEC3G complex formation," Journal of Experimental Medicine, vol. 203, no. 4, pp. 865-870, 2006.

[10] S. G. Conticello, C. J. F. Thomas, S. K. Petersen-Mahrt, and M. S. Neuberger, "Evolution of the AID/APOBEC family of polynucleotide (deoxy)cytidine deaminases," Molecular Biology and Evolution, vol. 22, no. 2, pp. 367-377, 2005.

[11] A. Jarmuz, A. Chester, J. Bayliss et al., "An anthropoidspecific locus of orphan C to U RNA-editing enzymes on chromosome 22," Genomics, vol. 79, no. 3, pp. 285-296, 2002.

[12] I. A. Maksakova, M. T. Romanish, L. Gagnier, C. A. Dunn, L. N. Van de Lagemaat, and D. L. Mager, "Retroviral elements and their hosts: Insertional mutagenesis in the mouse germ line," PLoS Genetics, vol. 2, no. 1, article e2, 2006.

[13] S. L. Sawyer, M. Emerman, and H. S. Malik, "Ancient adaptive evolution of the primate antiviral DNA-editing enzyme APOBEC3G," PLoS Biology, vol. 2, no. 9, article E275, 2004.

[14] R. S. Harris and M. T. Liddament, "Retroviral restriction by APOBEC proteins," Nature Reviews Immunology, vol. 4, no. 11, pp. 868-877, 2004.

[15] B. H.-J. Chang and L. Chan, "Evolutionary analysis of RNA editing enzymes," Methods, vol. 15, no. 1, pp. 41-50, 1998.

[16] H. Huthoff and M. H. Malim, "Cytidine deamination and resistance to retroviral infection: towards a structural understanding of the APOBEC proteins," Virology, vol. 334, no. 2, pp. 147-153, 2005.

[17] R. P. Bennett, E. Diner, M. P. Sowden, J. A. Lees, J. E. Wedekind, and H. C. Smith, "APOBEC-1 and AID are nucleo-cytoplasmic trafficking proteins but APOBEC3G cannot traffic," Biochemical and Biophysical Research Communications, vol. 350, no. 1, pp. 214-219, 2006.

[18] D. M. Lehmann, C. A. Galloway, C. MacElrevey, M. P. Sowden, J. E. Wedekind, and H. C. Smith, "Functional characterization of APOBEC-1 complementation factor phosphorylation sites," Biochimica et Biophysica Acta, vol. 1773, no. 3, pp. 408-418, 2007.

[19] H. C. Smith, A. Bottaro, M. P. Sowden, and J. E. Wedekind, "Activation induced deaminase: the importance of being specific," Trends in Genetics, vol. 20, no. 6, pp. 224-227, 2004.

[20] B. Teng, C. F. Burant, and N. O. Davidson, "Molecular cloning of an apolipoprotein B messenger RNA editing protein," Science, vol. 260, no. 5115, pp. 1816-1818, 1993.

[21] J. E. Wedekind, G. S. C. Dance, M. P. Sowden, and H. C. Smith, "Messenger RNA editing in mammals: new members of the APOBEC family seeking roles in the family business," Trends in Genetics, vol. 19, no. 4, pp. 207-216, 2003.

[22] J. E. Wedekind, R. Gillilan, A. Janda et al., "Nanostructures of APOBEC3G support a hierarchical assembly model of high molecular mass ribonucleoprotein particles from dimeric subunits," The Journal of Biological Chemistry, vol. 281, no. 50, pp. 38122-38126, 2006.

[23] K. N. Bishop, R. K. Holmes, A. M. Sheehy, N. O. Davidson, S.J. Cho, and M. H. Malim, "Cytidine deamination of retroviral DNA by diverse APOBEC proteins," Current Biology, vol. 14, no. 15, pp. 1392-1396, 2004.

[24] C. Rösler, J. Köck, M. H. Malim, H. E. Blum, and F. Weizsäcker, "Comment on "Inhibition of hepatitis B virus replication by APOBEC3G'”' Science, vol. 305, no. 5689, pp. 1403-1403, 2004.

[25] P. Turelli, B. Mangeat, S. Jost, S. Vianin, and D. Trono, "Inhibition of hepatitis B virus replication by APOBEC3G," Science, vol. 303, no. 5665, p. 1829, 2004.

[26] Y.-L. Chiu and W. C. Greene, "The APOBEC3 cytidine deaminases: an innate defensive network opposing exogenous retroviruses and endogenous retroelements," Annual Review of Immunology, vol. 26, pp. 317-353, 2008.

[27] H. P. Bogerd, H. L. Wiegand, B. P. Doehle, and B. R. Cullen, "The intrinsic antiretroviral factor APOBEC3B contains two enzymatically active cytidine deaminase domains," Virology, vol. 364, no. 2, pp. 486-493, 2007.

[28] Y. Dang, X. Wang, W. J. Esselman, and Y.-H. Zheng, "Identification of APOBEC3DE as another antiretroviral factor from the human APOBEC family," Journal of Virology, vol. 80, no. 21, pp. 10522-10533, 2006.

[29] B. P. Doehle, A. Schäfer, H. L. Wiegand, H. P. Bogerd, and B. R. Cullen, "Differential sensitivity of murine leukemia virus to APOBEC3-mediated inhibition is governed by virion exclusion," Journal of Virology, vol. 79, no. 13, pp. 8201-8207, 2005.

[30] K. Bourara, T. J. Liegler, and R. M. Grant, "Target cell APOBEC3C can induce limited G-to-A mutation in HIV-1," PLoS Pathogens, vol. 3, no. 10, article e153, 2007.

[31] Y. Dang, L. M. Siew, X. Wang, Y. Han, R. Lampen, and Y.H. Zheng, "Human cytidine deaminase APOBEC3H restricts HIV-1 replication," The Journal of Biological Chemistry, vol. 283, no. 17, pp. 11606-11614, 2008.

[32] A. Harari, M. Ooms, L. C. F. Mulder, and V. Simon, "Polymorphisms and splice variants influence the antiretroviral activity of Human APOBEC3H," Journal of Virology, vol. 83, no. 1, pp. 295-303, 2009.

[33] G. Peng, T. Greenwell-Wild, S. Nares et al., "Myeloid differentiation and susceptibility to HIV-1 are linked to APOBEC3 expression," Blood, vol. 110, no. 1, pp. 393-400, 2007.

[34] S. Cen, F. Guo, M. Niu, J. Saadatmand, J. Deflassieux, and L. Kleiman, "The interaction between HIV-1 gag and APOBEC3G," The Journal of Biological Chemistry, vol. 279, no. 32, pp. 33177-33184, 2004.

[35] K. Luo, B. Liu, Z. Xiao et al., "Amino-terminal region of the human immunodeficiency virus type 1 nucleocapsid is required for human APOBEC3G packaging," Journal of Virology, vol. 78, no. 21, pp. 11841-11852, 2004.

[36] A. Schäfer, H. P. Bogerd, and B. R. Cullen, "Specific packaging of APOBEC3G into HIV-1 virions is mediated by the nucleocapsid domain of the gag polyprotein precursor," Virology, vol. 328, no. 2, pp. 163-168, 2004.

[37] J. A. Dutko, A. Schäfer, A. E. Kenny, B. R. Cullen, and M. J. Curcio, "Inhibition of a yeast LTR retrotransposon by human APOBEC3 cytidine deaminases," Current Biology, vol. 15, no. 7, pp. 661-666, 2005.

[38] R. Suspène, P. Sommer, M. Henry et al., "APOBEC3G is a single-stranded DNA cytidine deaminase and functions independently of HIV reverse transcriptase," Nucleic Acids Research, vol. 32, no. 8, pp. 2421-2429, 2004.

[39] Y. Yu, Z. Xiao, E. S. Ehrlich, X. Yu, and X.-F. Yu, "Selective assembly of HIV-1 Vif-Cul5-ElonginB-ElonginC E3 ubiquitin ligase complex through a novel SOCS box and upstream cysteines," Genes and Development, vol. 18, no. 23, pp. 28672872, 2004. 
[40] R. S. Harris, K. N. Bishop, A. M. Sheehy et al., "DNA deamination mediates innate immunity to retroviral infection," Cell, vol. 113, no. 6, pp. 803-809, 2003.

[41] B. Mangeat, P. Turelli, G. Caron, M. Friedli, L. Perrin, and D. Trono, "Broad antiretroviral defence by human APOBEC3G through lethal editing of nascent reverse transcripts," Nature, vol. 424, no. 6944, pp. 99-103, 2003.

[42] H. Zhang, B. Yang, R. J. Pomerantz, C. Zhang, S. C. Arunachalam, and L. Gao, "The cytidine deaminase CEM15 induces hypermutation in newly synthesized HIV-1 DNA," Nature, vol. 424, no. 6944, pp. 94-98, 2003.

[43] K. Shindo, A. Takaori-Kondo, M. Kobayashi, A. Abudu, K. Fukunaga, and T. Uchiyama, "The enzymatic activity of CEM15/Apobec-3G is essential for the regulation of the infectivity of HIV-1 virion but not a sole determinant of its antiviral activity," The Journal of Biological Chemistry, vol. 278, no. 45, pp. 44412-44416, 2003.

[44] E. N. C. Newman, R. K. Holmes, H. M. Craig et al., "Antiviral function of APOBEC3G can be dissociated from cytidine deaminase activity," Current Biology, vol. 15, no. 2, pp. 166170, 2005.

[45] M. Santa-Marta, F. A. Da Silva, A. M. Fonseca, and J. Goncalves, "HIV-1 Vif can directly inhibit apolipoprotein B mRNA-editing enzyme catalytic polypeptide-like 3Gmediated cytidine deamination by using a single amino acid interaction and without protein degradation," The Journal of Biological Chemistry, vol. 280, no. 10, pp. 8765-8775, 2005.

[46] E. Miyagi, S. Opi, H. Takeuchi et al., "Enzymatically active APOBEC3G is required for efficient inhibition of human immunodeficiency virus type 1," Journal of Virology, vol. 81, no. 24, pp. 13346-13353, 2007.

[47] R. K. Holmes, F. A. Koning, K. N. Bishop, and M. H. Malim, "APOBEC3F can inhibit the accumulation of HIV-1 reverse transcription products in the absence of hypermutation: comparisons with APOBEC3G," The Journal of Biological Chemistry, vol. 282, no. 4, pp. 2587-2595, 2007.

[48] Y. Yang, F. Guo, S. Cen, and L. Kleiman, "Inhibition of initiation of reverse transcription in HIV-1 by human APOBEC3F," Virology, vol. 365, no. 1, pp. 92-100, 2007.

[49] Y. Iwatani, D. S. B. Chan, F. Wang et al., "Deaminaseindependent inhibition of HIV-1 reverse transcription by APOBEC3G," Nucleic Acids Research, vol. 35, no. 21, pp. 7096-7108, 2007.

[50] X.-Y. Li, F. Guo, L. Zhang, L. Kleiman, and S. Cen, "APOBEC3G inhibits DNA strand transfer during HIV-1 reverse transcription," The Journal of Biological Chemistry, vol. 282, no. 44, pp. 32065-32074, 2007.

[51] K. N. Bishop, M. Verma, E.-Y. Kim, S. M. Wolinsky, and M. H. Malim, "APOBEC3G inhibits elongation of HIV-1 reverse transcripts," PLoS Pathogens, vol. 4, no. 12, Article ID e1000231, 2008.

[52] J. L. Anderson and T. J. Hope, "APOBEC3G restricts early HIV-1 replication in the cytoplasm of target cells," Virology, vol. 375, no. 1, pp. 1-12, 2008.

[53] J. L. Mbisa, R. Barr, J. A. Thomas et al., "Human immunodeficiency virus type $1 \mathrm{cDNAs}$ produced in the presence of APOBEC3G exhibit defects in plus-strand DNA transfer and integration," Journal of Virology, vol. 81, no. 13, pp. 70997110, 2007.

[54] R. S. Aguiar and B. M. Peterlin, "APOBEC3 proteins and reverse transcription," Virus Research, vol. 134, no. 1-2, pp. 74-85, 2008.
[55] K. Luo, T. Wang, B. Liu et al., "Cytidine deaminases APOBEC3G and APOBEC3F interact with human immunodeficiency virus type 1 integrase and inhibit proviral DNA formation," Journal of Virology, vol. 81, no. 13, pp. 72387248, 2007.

[56] S. G. Conticello, R. S. Harris, and M. S. Neuberger, "The Vif protein of HIV triggers degradation of the human antiretroviral DNA deaminase APOBEC3G," Current Biology, vol. 13, no. 22, pp. 2009-2013, 2003.

[57] A. M. Sheehy, N. C. Gaddis, and M. H. Malim, "The antiretroviral enzyme APOBEC3G is degraded by the proteasome in response to HIV-1 Vif," Nature Medicine, vol. 9, no. 11, pp. 1404-1407, 2003.

[58] M. Marin, K. M. Rose, S. L. Kozak, and D. Kabat, "HIV-1 Vif protein binds the editing enzyme APOBEC $3 \mathrm{G}$ and induces its degradation," Nature Medicine, vol. 9, no. 11, pp. 1398-1403, 2003.

[59] X. Yu, Y. Yu, B. Liu et al., "Induction of APOBEC3G ubiquitination and degradation by an HIV-1 Vif-Cul5-SCF complex," Science, vol. 302, no. 5647, pp. 1056-1060, 2003.

[60] K. Stopak, C. de Noronha, W. Yonemoto, and W. C. Greene, "HIV-1 Vif blocks the antiviral activity of APOBEC3G by impairing both its translation and intracellular stability," Molecular Cell, vol. 12, no. 3, pp. 591-601, 2003.

[61] A. Mehle, B. Strack, P. Ancuta, C. Zhang, M. McPike, and D. Gabuzda, "Vif Overcomes the innate antiviral activity of APOBEC3G by promoting its degradation in the ubiquitinproteasome pathway," The Journal of Biological Chemistry, vol. 279, no. 9, pp. 7792-7798, 2004.

[62] Y. Yu, Z. Xiao, E. S. Ehrlich, X. Yu, and X.-F. Yu, "Selective assembly of HIV-1 Vif-Cul5-ElonginB-ElonginC E3 ubiquitin ligase complex through a novel SOCS box and upstream cysteines," Genes and Development, vol. 18, no. 23, pp. 28672872, 2004.

[63] B. Liu, P. T. N. Sarkis, K. Luo, Y. Yu, and X.-F. Yu, "Regulation of Apobec3F and human immunodeficiency virus type 1 Vif by Vif-Cul5-ElonB/C E3 ubiquitin ligase," Journal of Virology, vol. 79, no. 15, pp. 9579-9587, 2005.

[64] Y. Dang, L. M. Siew, and Y.-H. Zheng, "APOBEC3G is degraded by the proteasomal pathway in a vif-dependent manner without being polyubiquitylated," The Journal of Biological Chemistry, vol. 283, no. 19, pp. 13124-13131, 2008.

[65] S. Kao, M. A. Khan, E. Miyagi, R. Plishka, A. Buckler-White, and K. Strebel, "The human immunodeficiency virus type 1 Vif protein reduces intracellular expression and inhibits packaging of APOBEC3G (CEM15), a cellular inhibitor of virus infectivity," Journal of Virology, vol. 77, no. 21, pp. 11398-11407, 2003.

[66] R. Mariani, D. Chen, B. Schröfelbauer et al., "Species-specific exclusion of APOBEC3G from HIV-1 virions by Vif," Cell, vol. 114, no. 1, pp. 21-31, 2003.

[67] V. Simon, V. Zennou, D. Murray, Y. Huang, D. D. Ho, and P. D. Bieniasz, "Natural variation in vif: differential impact on APOBEC3G/3F and a potential role in HIV-1 diversification," PLoS Pathogens, vol. 1, no. 1, article e6, 2005.

[68] B. Schröfelbauer, T. Senger, G. Manning, and N. R. Landau, "Mutational alteration of human immunodeficiency virus type 1 vif allows for functional interaction with nonhuman primate APOBEC3G," Journal of Virology, vol. 80, no. 12, pp. 5984-5991, 2006.

[69] C. Tian, X. Yu, W. Zhang, T. Wang, R. Xu, and X.-F. $\mathrm{Yu}$, "Differential requirement for conserved tryptophans in 
human immunodeficiency virus type 1 vif for the selective suppression of APOBEC3G and APOBEC3F," Journal of Virology, vol. 80, no. 6, pp. 3112-3115, 2006.

[70] I. Paul, J. Cui, and E. L. Maynard, "Zinc binding to the HCCH motif of HIV-1 virion infectivity factor induces a conformational change that mediates protein-protein interactions," Proceedings of the National Academy of Sciences of the United States of America, vol. 103, no. 49, pp. 18475-18480, 2006.

[71] J. Goncalves, P. Jallepalli, and D. H. Gabuzda, "Subcellular localization of the Vif protein of human immunodeficiency virus type 1," Journal of Virology, vol. 68, no. 2, pp. 704-712, 1994.

[72] X.-Y. Ma, P. Sova, W. Chao, and D. J. Volsky, "Cysteine residues in the Vif protein of human immunodeficiency virus type 1 are essential for viral infectivity," Journal of Virology, vol. 68 , no. 3, pp. 1714-1720, 1994.

[73] A. Mehle, J. Goncalves, M. Santa-Marta, M. McPike, and D. Gabuzda, "Phosphorylation of a novel SOCS-box regulates assembly of the HIV-1 Vif-Cul5 complex that promotes APOBEC3G degradation," Genes and Development, vol. 18, no. 23, pp. 2861-2866, 2004.

[74] A. Mehle, E. R. Thomas, K. S. Rajendran, and D. Gabuzda, "A zinc-binding region in Vif binds Cul5 and determines cullin selection," The Journal of Biological Chemistry, vol. 281, no. 25, pp. 17259-17265, 2006.

[75] M. Kobayashi, A. Takaori-Kondo, Y. Miyauchi, K. Iwai, and T. Uchiyama, "Ubiquitination of APOBEC3G by an HIV-1 Vif-Cullin5-Elongin B-Elongin C complex is essential for Vif function," The Journal of Biological Chemistry, vol. 280, no. 19, pp. 18573-18578, 2005.

[76] K. Luo, Z. Xiao, E. Ehrlich et al., "Primate lentiviral virion infectivity factors are substrate receptors that assemble with cullin 5-E3 ligase through a HCCH motif to suppress APOBEC3G," Proceedings of the National Academy of Sciences of the United States of America, vol. 102, no. 32, pp. 1144411449, 2005.

[77] Z. Xiao, E. Ehrlich, K. Luo, Y. Xiong, and X.-F. Yu, "Zinc chelation inhibits HIV Vif activity and liberates antiviral function of the cytidine deaminase APOBEC3G," The FASEB Journal, vol. 21, no. 1, pp. 217-222, 2007.

[78] H. P. Bogerd, B. P. Doehle, H. L. Wiegand, and B. R. Cullen, "A single amino acid difference in the host APOBEC3G protein controls the primate species specificity of HIV type 1 virion infectivity factor," Proceedings of the National Academy of Sciences of the United States of America, vol. 101, no. 11, pp. 3770-3774, 2004.

[79] B. Mangeat, P. Turelli, S. Liao, and D. Trono, "A single amino acid determinant governs the species-specific sensitivity of APOBEC3G to Vif action," The Journal of Biological Chemistry, vol. 279, no. 15, pp. 14481-14483, 2004.

[80] B. Schröfelbauer, D. Chen, and N. R. Landau, "A single amino acid of APOBEC3G controls its species-specific interaction with virion infectivity factor (Vif)," Proceedings of the National Academy of Sciences of the United States of America, vol. 101, no. 11, pp. 3927-3932, 2004.

[81] H. Xu, E. S. Svarovskaia, R. Barr et al., "A single amino acid substitution in human APOBEC3G antiretroviral enzyme confers resistance to HIV-1 virion infectivity factor-induced depletion," Proceedings of the National Academy of Sciences of the United States of America, vol. 101, no. 15, pp. 5652-5657, 2004.

[82] H. Huthoff and M. H. Malim, "Identification of amino acid residues in APOBEC3G required for regulation by human immunodeficiency virus type 1 Vif and virion encapsidation," Journal of Virology, vol. 81, no. 8, pp. 38073815, 2007.

[83] L. Zhang, J. Saadatmand, X. Li et al., "Function analysis of sequences in human APOBEC3G involved in Vif-mediated degradation," Virology, vol. 370, no. 1, pp. 113-121, 2008.

[84] R. A. Russell, J. Smith, R. Barr, D. Bhattacharyya, and V. K. Pathak, "Distinct domains within APOBEC3G and APOBEC3F interact with separate regions of human immunodeficiency virus type 1 Vif," Journal of Virology, vol. 83, no. 4, pp. 1992-2003, 2009.

[85] Y. Iwatani, D. S. B. Chan, L. Liu et al., "HIV-1 Vif-mediated ubiquitination/degradation of APOBEC3G involves four critical lysine residues in its C-terminal domain," Proceedings of the National Academy of Sciences of the United States of America, vol. 106, no. 46, pp. 19539-19544, 2009.

[86] R. A. Russell and V. K. Pathak, "Identification of two distinct human immunodeficiency virus type 1 vif determinants critical for interactions with human APOBEC3G and APOBEC3F," Journal of Virology, vol. 81, no. 15, pp. 82018210, 2007.

[87] A. Mehle, H. Wilson, C. Zhang et al., "Identification of an APOBEC3G binding site in human immunodeficiency virus type 1 Vif and inhibitors of Vif-APOBEC3G binding," Journal of Virology, vol. 81, no. 23, pp. 13235-13241, 2007.

[88] Z. He, W. Zhang, G. Chen, R. Xu, and X.-F. Yu, "Characterization of Conserved Motifs in HIV-1 Vif Required for APOBEC3G and APOBEC3F Interaction," Journal of Molecular Biology, vol. 381, no. 4, pp. 1000-1011, 2008.

[89] E. Pery, K. S. Rajendran, A. J. Brazier, and D. Gabuzda, "Regulation of APOBEC3 proteins by a Novel YXXL motif in human immunodeficiency virus type 1 vif and simian immunodeficiency virus SIVagm vif," Journal of Virology, vol. 83, no. 5, pp. 2374-2381, 2009.

[90] T. Yamashita, K. Kamada, K. Hatcho, A. Adachi, and M. Nomaguchi, "Identification of amino acid residues in HIV1 Vif critical for binding and exclusion of APOBEC3G/F," Microbes and Infection, vol. 10, no. 10-11, pp. 1142-1149, 2008.

[91] G. Chen, Z. He, T. Wang, R. Xu, and X.-F. Yu, "A patch of positively charged amino acids surrounding the human immunodeficiency virus type 1 Vif SLVx4Yx9Y motif influences its interaction with APOBEC3G," Journal of Virology, vol. 83, no. 17, pp. 8674-8682, 2009.

[92] Y. Dang, X. Wang, T. Zhou, I. A. York, and Y.-H. Zheng, "Identification of a novel WXSLVK motif in the $\mathrm{N}$ terminus of human immunodeficiency virus and simian immunodeficiency virus Vif that is critical for APOBEC3G and APOBEC3F neutralization," Journal of Virology, vol. 83, no. 17, pp. 8544-8552, 2009.

[93] K. Shirakawa, A. Takaori-Kondo, M. Yokoyama et al., "Phosphorylation of APOBEC3G by protein kinase A regulates its interaction with HIV-1 Vif," Nature Structural and Molecular Biology, vol. 15, no. 11, pp. 1184-1191, 2008.

[94] M. Fujita, A. Sakurai, A. Yoshida et al., "Amino acid residues 88 and 89 in the central hydrophilic region of human immunodeficiency virus type 1 Vif are critical for viral infectivity by enhancing the steady-state expression of Vif," Journal of Virology, vol. 77, no. 2, pp. 1626-1632, 2003.

[95] M. Douaisi, S. Dussart, M. Courcoul et al., "The tyrosine kinases Fyn and Hck favor the recruitment of tyrosinephosphorylated APOBEC3G into vif-defective HIV-1 particles," Biochemical and Biophysical Research Communications, vol. 329, no. 3, pp. 917-924, 2005. 
[96] S. Yang, Y. Sun, and H. Zhang, "The multimerization of human immunodeficiency virus type I Vif protein: a requirement for Vif function in the viral life cycle," The Journal of Biological Chemistry, vol. 276, no. 7, pp. 48894893, 2001.

[97] J. H. Miller, V. Presnyak, and H. C. Smith, "The dimerization domain of HIV-1 viral infectivity factor Vif is required to block virion incorporation of APOBEC3G," Retrovirology, vol. 4, article 81, 2007.

[98] G. Hassaïne, M. Courcoul, G. Bessou et al., "The tyrosine Kinase Hck is an inhibitor of HIV-1 replication counteracted by the viral Vif protein," The Journal of Biological Chemistry, vol. 276, no. 20, pp. 16885-16893, 2001.

[99] B. Yang, L. Gao, L. Li et al., "Potent suppression of viral infectivity by the peptides that inhibit multimerization of human immunodeficiency virus type 1 (HIV-1) Vif proteins," The Journal of Biological Chemistry, vol. 278, no. 8, pp. 65966602, 2003.

[100] J. P. Donahue, M. L. Vetter, N. A. Mukhtar, and R. T. D'Aquila, "The HIV-1 Vif PPLP motif is necessary for human APOBEC3G binding and degradation," Virology, vol. 377, no. 1, pp. 49-53, 2008.

[101] D. J. Hazuda, P. Felock, M. Witmer et al., "Inhibitors of strand transfer that prevent integration and inhibit HIV-1 replication in cells," Science, vol. 287, no. 5453, pp. 646-650, 2000.

[102] C. Pannecouque, W. Pluymers, B. Maele et al., "New class of HIV integrase inhibitors that block viral replication in cell culture," Current Biology, vol. 12, no. 14, pp. 1169-1177, 2002.

[103] M. Witvrouw, C. Pannecouque, W. M. Switzer, T. M. Folks, E. De Clercq, and W. Heneine, "Susceptibility of HIV-2, SIV and SHIV to various anti-HIV-1 compounds: implications for treatment and postexposure prophylaxis," Antiviral Therapy, vol. 9, no. 1, pp. 57-65, 2004.

[104] C. T. Wild, D. C. Shugars, T. K. Greenwell, C. B. McDanal, and T. J. Matthews, "Peptides corresponding to a predictive $\alpha$-helical domain of human immunodeficiency virus type 1 gp41 are potent inhibitors of virus infection," Proceedings of the National Academy of Sciences of the United States of America, vol. 91, no. 21, pp. 9770-9774, 1994.

[105] S. Kao, R. Goila-Gaur, E. Miyagi et al., "Production of infectious virus and degradation of APOBEC3G are separable functional properties of human immunodeficiency virus type 1 Vif," Virology, vol. 369, no. 2, pp. 329-339, 2007.

[106] A. Jarmuz, A. Chester, J. Bayliss et al., "An anthropoidspecific locus of orphan C to U RNA-editing enzymes on chromosome 22," Genomics, vol. 79, no. 3, pp. 285-296, 2002.

[107] J. Li, M. J. Potash, and D. J. Volsky, "Functional domains of APOBEC3G required for antiviral activity," Journal of Cellular Biochemistry, vol. 92, no. 3, pp. 560-572, 2004.

[108] F. Navarro, B. Bollman, H. Chen et al., "Complementary function of the two catalytic domains of APOBEC3G," Virology, vol. 333, no. 2, pp. 374-386, 2005.

[109] W. Lv, Z. Liu, H. Jin, X. Yu, L. Zhang, and L. Zhang, "Three-dimensional structure of HIV-1 VIF constructed by comparative modeling and the function characterization analyzed by molecular dynamics simulation," Organic and Biomolecular Chemistry, vol. 5, no. 4, pp. 617-626, 2007.

[110] K. Stopak and W. C. Greene, "Protecting APOBEC3G: a potential new target for HIV drug discovery," Current Opinion in Investigational Drugs, vol. 6, no. 2, pp. 141-147, 2005.
[111] W. J. Fessel, "A new approach to an AIDS vaccine: creating antibodies to HIV vif will enable apobec3G to turn HIVinfection into a benign problem," Medical Hypotheses, vol. 64, no. 2, pp. 261-263, 2005.

[112] M. Mezei and J. Minarovits, "Reversal of HIV drug resistance and novel strategies to curb HIV infection: the viral infectivity factor Vif as a target and tool of therapy," Current Drug Targets, vol. 7, no. 7, pp. 881-885, 2006.

[113] J. M. Carr, A. J. Davis, F. Feng, C. J. Burrell, and P. Li, "Cellular interactions of virion infectivity factor (Vif) as potential therapeutic targets: APOBEC3G and more?" Current Drug Targets, vol. 7, no. 12, pp. 1583-1593, 2006.

[114] R. Nathans, H. Cao, N. Sharova et al., "Small-molecule inhibition of HIV-1 Vif," Nature Biotechnology, vol. 26, no. 10, pp. 1187-1192, 2008.

[115] S. Opi, S. Kao, R. Goila-Gaur et al., "Human immunodeficiency virus type 1 Vif inhibits packaging and antiviral activity of a degradation-resistant APOBEC3G variant," Journal of Virology, vol. 81, no. 15, pp. 8236-8246, 2007.

[116] B. Berkhout and A. de Ronde, "APOBEC3G versus reverse transcriptase in the generation of HIV-1 drug-resistance mutations," AIDS, vol. 18, no. 13, pp. 1861-1863, 2004.

[117] K. N. Bishop, R. K. Holmes, and M. H. Malim, "Antiviral potency of APOBEC proteins does not correlate with cytidine deamination," Journal of Virology, vol. 80, no. 17, pp. 84508458, 2006.

[118] V. B. Soros, W. Yonemoto, and W. C. Greene, "Newly synthesized APOBEC3G is incorporated into HIV virions, inhibited by HIV RNA, and subsequently activated by RNase H," PLoS Pathogens, vol. 3, no. 2, article e15, 2007. 

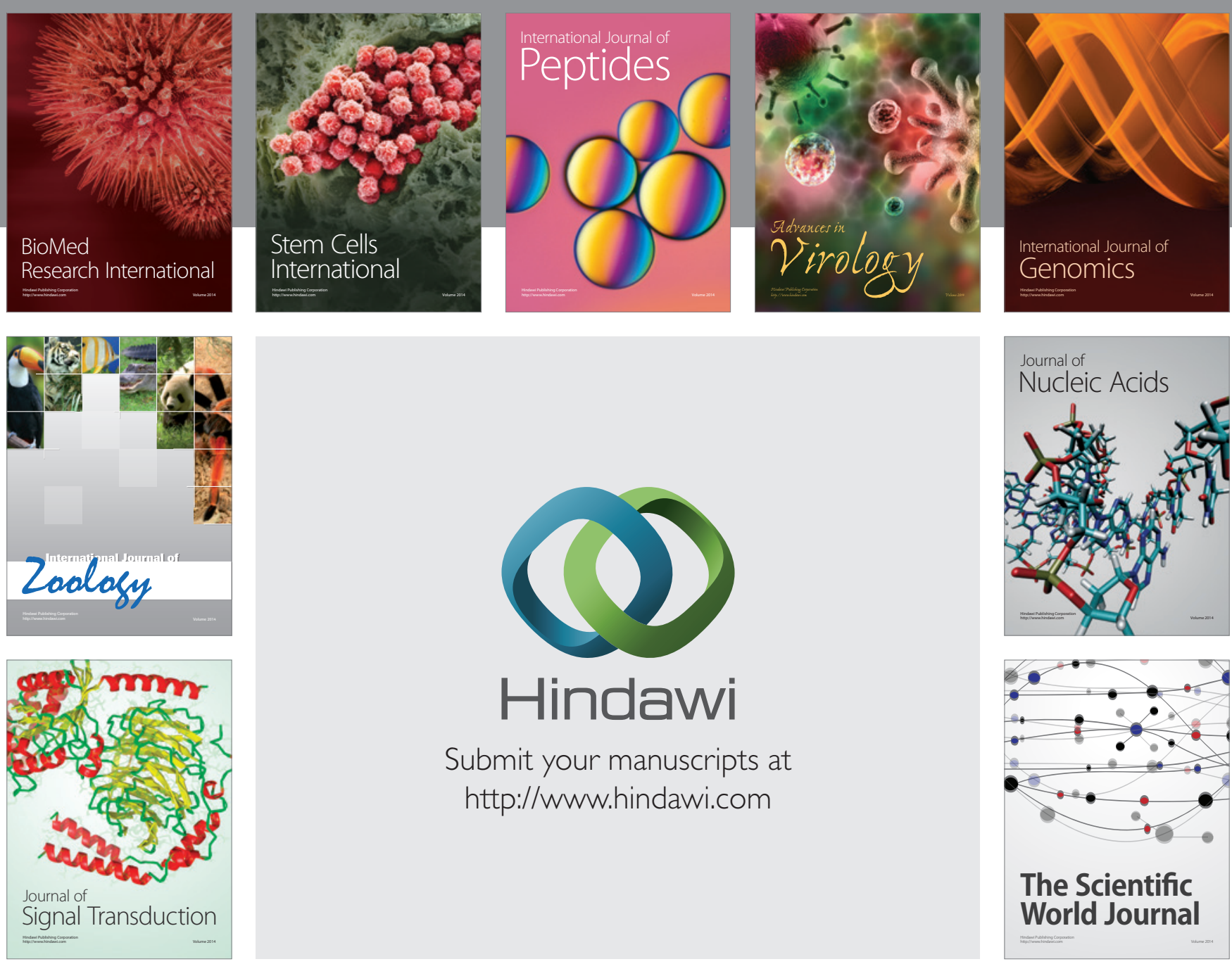

Submit your manuscripts at

http://www.hindawi.com
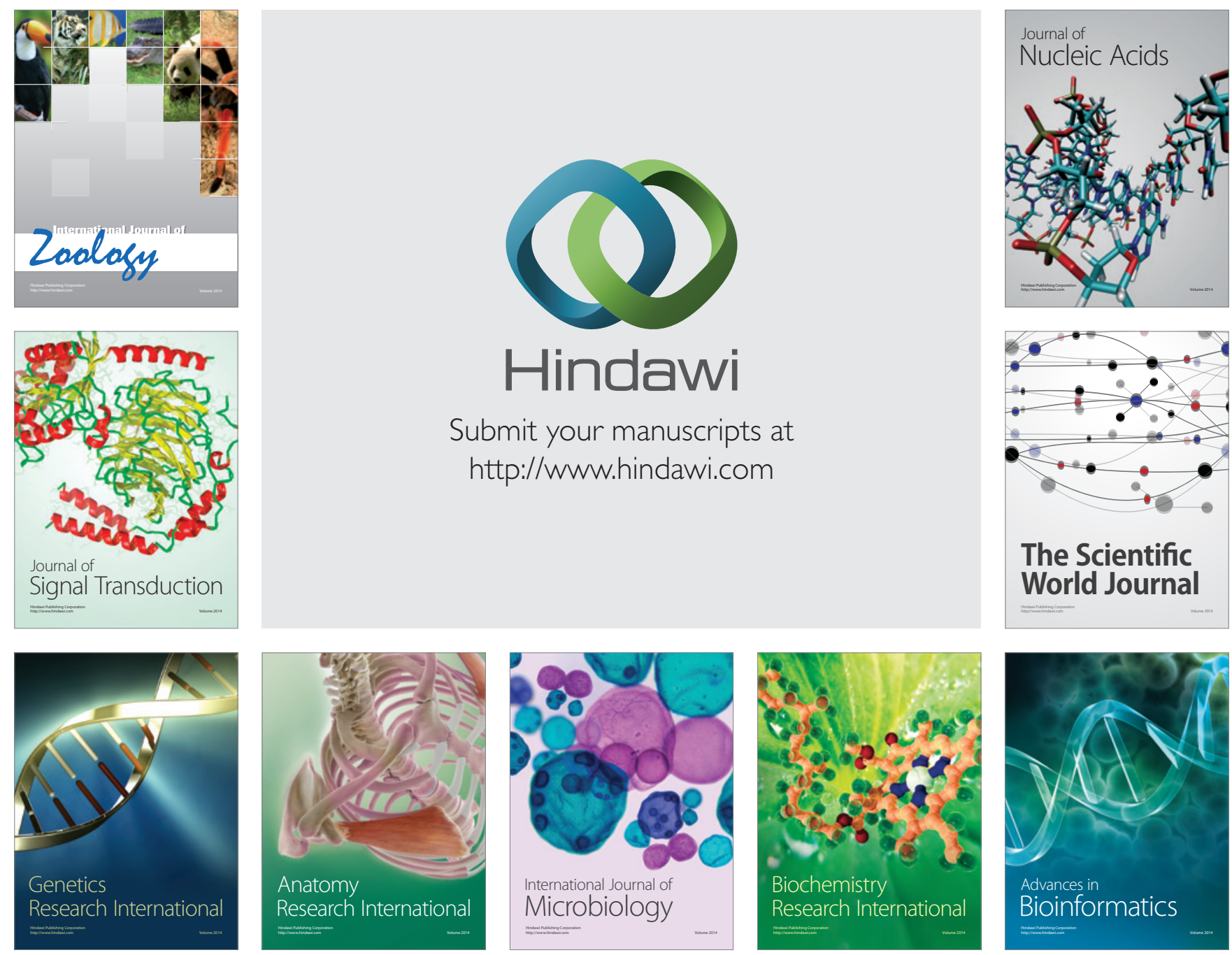

The Scientific World Journal
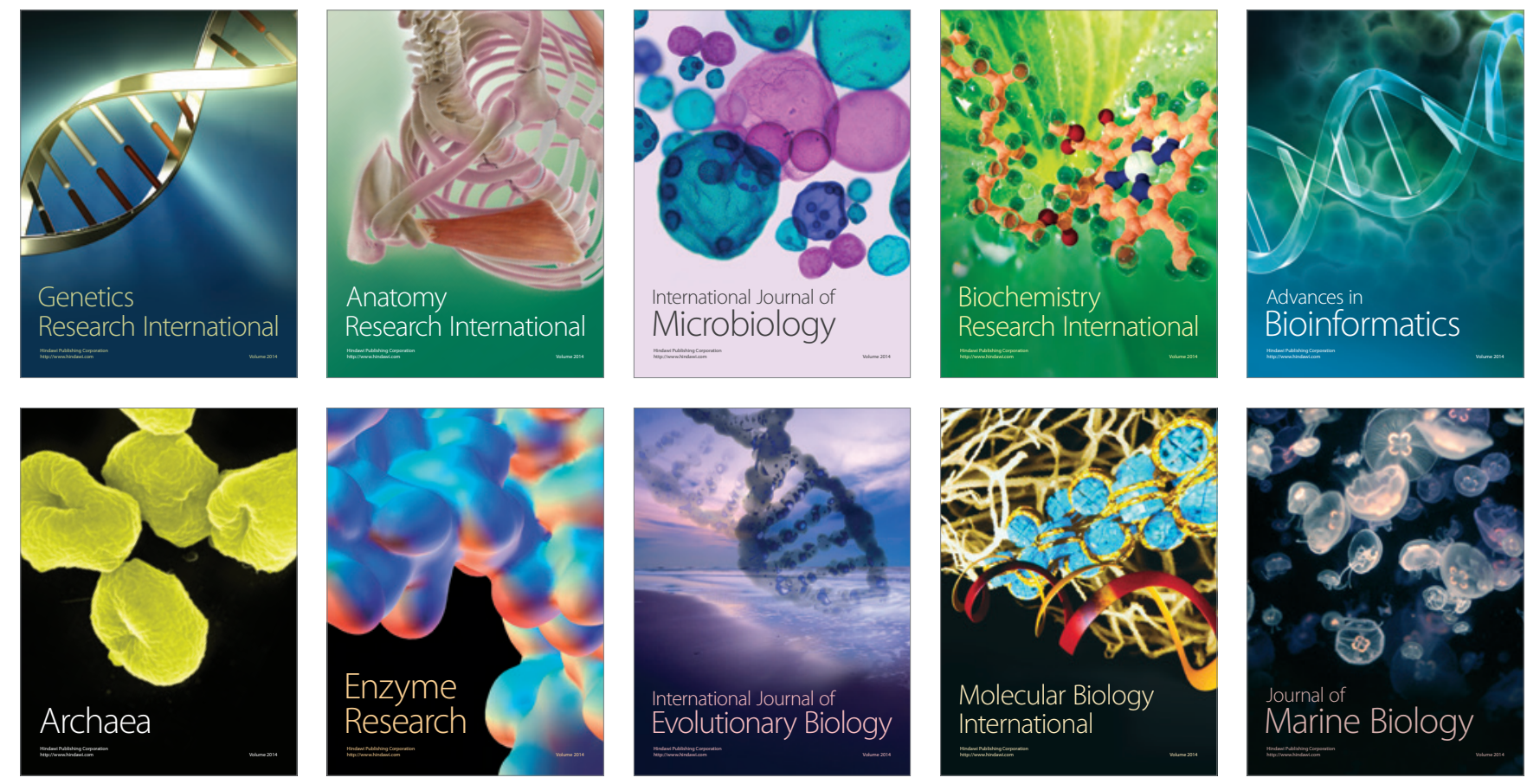